This item was submitted to Loughborough's Research Repository by the author.

Items in Figshare are protected by copyright, with all rights reserved, unless otherwise indicated.

\title{
Finiteness results for 3-folds with semiample anticanonical bundle
}

PLEASE CITE THE PUBLISHED VERSION

http://dx.doi.org/10.4310/MRL.2015.v22.n2.a11

\section{PUBLISHER}

International Press

\section{VERSION}

VoR (Version of Record)

\section{PUBLISHER STATEMENT}

This work is made available according to the conditions of the Creative Commons Attribution-NonCommercialNoDerivatives 4.0 International (CC BY-NC-ND 4.0) licence. Full details of this licence are available at: https://creativecommons.org/licenses/by-nc-nd/4.0/

\section{LICENCE}

CC BY-NC-ND 4.0

\section{REPOSITORY RECORD}

Prendergast-Smith, Artie. 2019. "Finiteness Results for 3-folds with Semiample Anticanonical Bundle". figshare. https://hdl.handle.net/2134/17285. 


\title{
Finiteness results for 3-folds with semiample anticanonical bundle
}

\author{
Artie Prendergast-Smith
}

The purpose of this paper is to give some evidence for the MorrisonKawamata cone conjecture for klt pairs. Roughly speaking, the cone conjecture predicts that in appropriate 'Calabi-Yau-type' situations, the groups of automorphisms and pseudo-automorphisms of a projective variety act with rational polyhedral fundamental domain on the nef and movable cones of the variety. (See Section 1 for the precise statement.)

In this paper we prove some statements in this direction, in the case of a mildly singular 3-fold with semiample anticanonical bundle of positive Iitaka dimension. Let us say a bit about how such a 3-fold looks geometrically. The anticanonical bundle defines a contraction morphism $X \rightarrow S$ to a positive-dimensional base; by adjunction all smooth fibres are varieties whose canonical bundle is torsion. So the generic fibre is a point, an elliptic curve, or a Calabi-Yau surface, according as the Iitaka dimension is 3, 2, or 1. (Here a Calabi-Yau surface means an abelian, K3, Enriques or hyperelliptic surface.) If the contraction morphism is equidimensional, classification results due to Kodaira and Miranda (for fibre dimension 1) and Kulikov and Crauder-Morrison (for fibre dimension 2) give information about the singular fibres. (See [5] for details of these classification results.)

We will see that all 3 -folds of this kind fall inside the scope of the Morrison-Kawamata cone conjecture. Our main result is the following finiteness theorem, which can be regarded as a weak form of the conjecture for these varieties. (All varieties are assumed projective, over an algebraically closed field of characteristic zero.)

Theorem 0.1. Let $X$ be a Q-factorial terminal Gorenstein 3-fold with $-K_{X}$ semiample of positive Iitaka dimension. Let $f: X \rightarrow S$ be the contraction morphism given by sections of some power of $-K_{X}$. Then the effective movable cone $\overline{M(X)}$ decomposes as the union of the effective nef cones of the small Q-factorial modifications of $X$, all of these small Q-factorial modifications are over $S$, and the decomposition is finite up to the action of the group PsAut $(X / S)$ of pseudo-automorphisms of $X$ over $S$. 
(For this statement, a pseudo-automorphism of $X$ is a birational self-map of $X$ that contracts no divisors, and $\operatorname{PsAut}(X / S)$ is the group of pseudoautomorphisms $\phi$ of $X$ such that $f \circ \phi=f$ as rational maps. See Section 1 for details.)

For terminal 3-folds, Gorenstein singularities are the same thing as hypersurface singularities. The latter description is geometrically clearer, but we use the former description to remain consistent with the literature. In any case, we make the Gorenstein assumption so that we can use a theorem of Mori and its extension by Cutkosky classifying certain contraction morphisms on terminal 3 -folds.

We will explain in Section 1 why Theorem 0.1 can be regarded as a weak form of the cone conjecture for the given class of varieties. The key ingredients in the proof of the theorem are Kawamata's corresponding result for the relative movable cone $\overline{M(X / S)}^{e}$ and the Mori-Cutkosky classification result mentioned above. Indeed, given these two results, our theorem follows as a simple consequence. The proof is given in Section 2 .

In Section 4 we study the movable cone in more detail, under the stronger assumption that $-K_{X}$ has Iitaka dimension 2 and the contraction $X \rightarrow$ $S$ is equidimensional. In particular we get a complete description of the relative movable cone (Theorem 4.6), which leads to a finiteness result for the (absolute) movable cone (Theorem 4.10) and a proof of the full movable cone conjecture in some examples (Corollary 4.11 and Example 4.12).

In Section 5 we give some partial results concerning the conjecture on the nef cone. In particular, for the simple case of smooth non-rationally connected $X$ such that $-K_{X}$ is semiample of Iitaka dimension 2, we show how the nef cone conjecture follows from classification results of BauerPeternell (Theorem 5.6).

\section{The cone conjecture}

We work throughout over an algebraically closed field $k$ of characteristic 0 .

Suppose $f: X \rightarrow Z$ is a projective surjective morphism of normal varieties with connected fibres. A Cartier divisor $D$ on $X$ is said to be nef over $Z$ (resp. movable over $Z$, big over $Z$, effective over $Z$ ) if $D \cdot C \geq 0$ for all curves $C$ mapped to a point by $f$ (resp. if codim $\operatorname{Supp} \operatorname{Coker}\left(f^{*} f_{*} \mathcal{O}_{X}(D) \rightarrow\right.$ $\left.\mathcal{O}_{X}(D)\right) \geq 2$, if the Iitaka dimension $\kappa\left(X_{\eta}, D_{\eta}\right)=\operatorname{dim} X-\operatorname{dim} Z$ for $\eta \in Z$ the generic point, if $\left.f_{*} \mathcal{O}_{X}(D) \neq 0\right)$.

We define the real vector space $N^{1}(X / Z)$ to be $\left(\operatorname{Div}(X) / \equiv_{Z}\right) \otimes \mathbf{R}$ where $\operatorname{Div}(X)$ is the group of Cartier divisors on $X$ and $\equiv_{Z}$ denotes numerical equivalence over $Z$. The relative nef cone $\overline{A(X / Z)}$ (resp. closed relative 
movable cone $\overline{M(X / Z)}$ ) is the closed convex cone generated by classes of Cartier divisors which are nef over $Z$ (resp. movable over $Z$ ). The relative big cone $B(X / Z)$ is the open cone generated by Cartier divisors which are big over $Z$. The relative effective cone $B^{e}(X / Z)$ is the cone generated by Cartier divisors which are effective over $Z$. We denote by $\overline{A(X / Z)}^{e}$ and $\overline{M(X / Z)})^{e}$ the intersections $\overline{A(X / Z)} \cap B^{e}(X / Z)$ and $M(X / Z) \cap B^{e}(X / Z)$, and call them the relative effective nef cone and relative effective movable cone respectively.

For later use, we also make the following definitions. The real vector space $N_{1}(X / Z)$ is defined to be the dual of $N^{1}(X / Z)$; equivalently, it is the real vector space spanned by numerical classes of curves on $X$ which map to a point on $Z$. We define the relative closed cone of curves, denoted $\overline{\operatorname{Curv}(X / Z)}$, to be the closed convex cone in $N_{1}(X / Z)$ dual to the cone $\overline{A(X / Z)}$. (Equivalently, it is the closed convex cone spanned by the classes of irreducible curves on $X$ which map to a point on $Z$.) We should stress that our notation for the cone of curves is nonstandard: it replaces the standard notation $\overline{N E(X / Z)}$. The new notation is intended to be more informative.

For all the notation introduced above, in the case that $Z=$ Spec $k$ we will omit it from the notation and simply write $N^{1}(X), \overline{A(X)}$, and so on. In this case we also omit the adjective 'relative' from all the corresponding terms.

Define a pseudo-isomorphism from $X_{1}$ to $X_{2}$ over $Z$ to be a birational map $X_{1} \rightarrow X_{2}$ over $Z$ which is an isomorphism in codimension 1. For a $\mathbf{Q}-$ factorial variety $X$ over $Z$, a small $\mathbf{Q}$-factorial modification (SQM) of $X$ over $Z$ means a pseudo-isomorphism over $Z$ from $X$ to another $\mathbf{Q}$-factorial variety with a projective morphism to $Z$. Note that if $\alpha: X^{\prime} \rightarrow-\rightarrow X$ is an SQM over $Z$, there is a canonical identification $N^{1}\left(X^{\prime} / Z\right) \cong N^{1}(X / Z)$ given by proper transform of divisors. This identification maps the effective movable cone of $X^{\prime}$ to that of $X$, so in particular identifies the effective nef cone $\overline{A\left(X^{\prime} / Z\right)}{ }^{e}$ with a subcone of $\overline{M(X / Z)}{ }^{e}$. This will explain the second statement of Conjecture 1.1. It is important to note that the identification depends on the map $\alpha$ and not just the variety $X^{\prime}$; we will write $\overline{A\left(X^{\prime} / Z, \alpha\right)}$ for the image of the nef cone inside $N^{1}(X / Z)$ when it is important to keep track of the identification.

For an $\mathbf{R}$-divisor $\Delta$ on a $\mathbf{Q}$-factorial variety $X$, the pair $(X, \Delta)$ is $k l t$ if for all resolutions $\pi: \tilde{X} \rightarrow X$ with a simple normal crossing $\mathbf{R}$-divisor $\tilde{\Delta}$ such that $K_{\tilde{X}}+\tilde{\Delta}=\pi^{*}\left(K_{X}+\Delta\right)$, the coefficients of $\tilde{\Delta}$ are less than 1 . (For instance if $X$ is smooth and $D$ is a smooth divisor on $X$, then $(X, r D)$ is klt for any $r<1$.) We say that $(X / Z, \Delta)$ is a klt Calabi-Yau pair if $(X, \Delta)$ 
is a Q-factorial klt pair with $\Delta$ effective such that $K_{X}+\Delta$ is numerically trivial over $Z$.

We denote the groups of automorphisms or pseudo-automorphisms of $X$ over $Z$ which preserve a divisor $\Delta$ by $\operatorname{Aut}(X / Z, \Delta)$ and $\operatorname{PsAut}(X / Z, \Delta)$. A rational polyhedral cone in $N^{1}(X / Z)$ means a convex cone spanned by a finite set of classes of Cartier divisors.

Conjecture 1.1. Let $(X / Z, \Delta)$ be a klt Calabi-Yau pair. Then:

(1) The number of $A u t(X / Z, \Delta)$-equivalence classes of faces of the relative effective nef cone $\overline{A(X / Z)}$ corresponding to birational contractions or fibre space structures is finite. Moreover, there exists a rational polyhedral cone $\Pi$ which is a fundamental domain for the action of $A u t(X / Z, \Delta)$ on $\overline{A(X / Z)}^{e}$ in the sense that

(a) $\overline{A(X / Z)}^{e}=\operatorname{Aut}(X / Z, \Delta) \cdot \Pi$,

(b) Int $\Pi \cap g_{*}$ Int $\Pi=\emptyset$ for any $g \in \operatorname{Aut}(X / Z, \Delta)$ such that $g_{*} \neq 1$ (where $g_{*} \in G L\left(N^{1}(X / Z)\right)$ is the linear automorphism induced by $\left.g\right)$.

(2) The number of PsAut $(X / Z, \Delta)$-equivalence classes of chambers ${\overline{A\left(X^{\prime} / Z, \alpha\right)}}^{e}$ in the relative effective movable cone $\overline{M(X / Z)}$ corresponding to $S Q M s \alpha: X^{\prime} \rightarrow X$ of $X$ over $Z$ is finite. Moreover, there exists a rational polyhedral cone $\Pi^{\prime}$ which is a fundamental domain for the action of PsAut $(X / Z, \Delta)$ on $\overline{M(X / Z)}$.

The first statement in parts (1) and (2) of the conjecture follows from the second. We refer to the first statement in parts (1) and (2) as the weak (nef or movable) cone conjecture.

Now we explain how the conjecture relates to Theorem 0.1. Suppose $X$ is a $\mathbf{Q}$-factorial terminal Gorenstein 3-fold with $-K_{X}$ semiample (meaning that for some positive integer $m$ the line bundle $-m K_{X}$ is basepoint-free) and of positive Iitaka dimension. Since $X$ is terminal Gorenstein, it has isolated singularities [25, Theorem 1.1]. Therefore choosing $m$ large enough so that $-m K_{X}$ is basepoint-free, a general divisor $D \in\left|-m K_{X}\right|$ does not intersect the singular locus of $X$. Moreover, Bertini's theorem says that a general $D \in\left|-m K_{X}\right|$ is smooth outside the singular locus of $X$, so by the previous sentence such a divisor $D$ must be smooth. Any log resolution of $X$ is then a log resolution of $(X, D)$, and on such a resolution the discrepancies of all exceptional divisors are the same for $(X, D)$ as for $(X, 0)$. Since $X$ is terminal, these are in particular positive. Assuming without loss of generality that $m>1$, and putting $\Delta=\frac{1}{m} D$, the pair $(X, \Delta)$ is therefore a klt pair with $\Delta$ effective, and $K_{X}+\Delta=0$ in $N^{1}(X)$. In other words $(X, \Delta)$ is a klt 
Calabi-Yau pair (over $Z=\operatorname{Spec} k$ ). So the weak movable cone conjecture predicts that there should be finitely many $\operatorname{PsAut}(X, \Delta)$-orbits of nef cones inside the effective movable cone $\overline{M(X)}^{e}$. Finally since we have a morphism $f: X \rightarrow S$ given by a multiple of the line bundle $-K_{X}$, the divisor $\Delta$ is a pullback of a $\mathbf{Q}$-divisor on $S$. Therefore the group $\operatorname{PsAut}(X / S)$ is a subgroup of $\operatorname{PsAut}(X, \Delta)$. Theorem 0.1 then implies that the weak movable cone conjecture holds for $(X, \Delta)$.

We conclude this section by outlining the history and current status of Conjecture 1.1. Inspired by mirror symmetry, Morrison [20] first proposed the conjecture for Calabi-Yau varieties. This was generalised to CalabiYau fibre spaces in [11] and to klt Calabi-Yau pairs in [29]. The conjecture was proved for Calabi-Yau surfaces by Sterk-Looijenga, Namikawa, and Kawamata [11, 21, 26], for 3-dimensional Calabi-Yau fibre spaces over a positive-dimensional base by Kawamata [11], and for klt Calabi-Yau pairs of dimension 2 by Totaro [29]. In dimension 3 the conjecture remains open, although there are significant results due to Oguiso-Peternell [22], Szendröi [27], Uehara [30], and Wilson [31], and verifications of special cases by Grassi-Morrison [7], Borcea [3], and Fryers [6]. More recently, the conjecture has been verified for abelian varieties [24], and for hyperkähler varieties many cases have been proved by Markman and Markman-Yoshioka [17, 18], using Verbitsky's Global Torelli theorem.

\section{Proof of Theorem 0.1}

In this section we give a proof of Theorem 0.1, thereby showing that the weak movable cone conjecture holds for $\mathbf{Q}$-factorial terminal Gorenstein 3folds with anticanonical bundle semiample of positive Iitaka dimension. As mentioned in the introduction, the proof follows easily from Kawamata's corresponding result in the relative case and Mori's classification of $K$-negative extremal 3-fold contractions. The basic idea is to prove that any non-nef effective movable divisor on our 3 -fold can be made nef by a sequence of flops, and that these flops are compatible with the morphism $f: X \rightarrow S$.

The first step is to use the the classification of $K$-negative extremal 3-fold contractions, due to Mori [19] and extended to the singular case by Cutkosky [4]. Here a Mori fibre space means a contraction morphism $f: X \rightarrow Z$ with $\operatorname{dim} Z<\operatorname{dim} X$, relative Picard number $\rho(X / Z)=1$, and $-K_{X}$ ample over $Z$. (For later use, we give the classification statement in more detail than we currently need.) 
Theorem 2.1 (Mori-Cutkosky). Suppose $X$ is a Q-factorial terminal Gorenstein 3-fold. Suppose $R \subset \overline{C u r v(X)}$ is $K$-negative extremal ray and $f: X \rightarrow Z$ is the contraction of $R$. Then either $\operatorname{dim} Z \leq 2$ and $f: X \rightarrow Z$ is a Mori fibre space, or else $f$ is birational, the exceptional set Exc $(f)$ is a prime divisor $D$ on $X$, and the possibilities for $D$ and $f$ are as follows:

(1) $f: X \rightarrow Z$ is the blowup of the ideal sheaf $I_{C}$ of a reduced irreducible lci curve $C \subset Z$ with exceptional divisor $D$;

(2) $D \cong \mathbf{P}^{2}$ with normal bundle $\mathcal{O}_{D}(D) \cong \mathcal{O}_{\mathbf{P}^{2}}(-1)$, and $f$ contracts $D$ to a smooth point;

(3) $D \cong \mathbf{P}^{1} \times \mathbf{P}^{1}$ with $\mathcal{O}_{D}(D)$ of bidegree $(-1,-1)$, and $f$ contracts $D$ to a point;

(4) $D$ is isomorphic to a singular quadric in $\mathbf{P}^{3}$ with $\mathcal{O}_{D}(D)=\mathcal{O}_{D} \otimes$ $\mathcal{O}_{\mathbf{P}^{3}}(-1)$, and $f$ contracts $D$ to a point;

(5) $D \cong \mathbf{P}^{2}$ with normal bundle $\mathcal{O}_{D}(D) \cong \mathcal{O}_{\mathbf{P}^{2}}(-2)$, and $f$ contracts $D$ to a point.

Moreover if $X$ is smooth, then in Case 1 above $Z$ is smooth, $C$ is a smooth curve in $Z$, and $D \rightarrow C$ is a $\mathbf{P}^{1}$-bundle.

Corollary 2.2. Suppose $X$ is a $\mathbf{Q}$-factorial terminal Gorenstein 3-fold and $x \in \overline{M(X)}$. Then $x \cdot R \geq 0$ for any $K$-negative extremal ray $R$ of $\overline{\operatorname{Curv}(X)}$.

Proof. By continuity and homogeneity it suffices to consider the case when $x=D$ is the class of a movable Cartier divisor. If $D \cdot R<0$ then any curve with class in $R$ must be contained in the base locus of $D$. But by the classification in Theorem 2.1, for any such $R$ the union of all curves with class in $R$ has codimension at most 1 in $X$, which contradicts the fact that $D$ is movable.

Now we can prove the decomposition of the movable cone into nef cones of SQMs. The key point is that all the SQMs $\alpha: X^{\prime} \rightarrow X$ we obtain are over $S$ : that is, they come with a morphism $f^{\prime}: X^{\prime} \rightarrow S$ such that $f^{\prime}=f \circ \alpha$. Our proof of the theorem is very similar to that of Kawamata [11] for the case of a Calabi-Yau fibre space.

Before stating the theorem we need some definitions. For a normal variety $Y$ and an R-Cartier divisor $D$ on $Y$, a $D$-flopping contraction is a proper birational morphism $f: Y \rightarrow Z$ to a normal variety $Z$ such that $\operatorname{Exc}(f)$ has codimension at least 2 in $Y$, the divisor $-\left(K_{Y}+D\right)$ is $\mathbf{R}$-Cartier and ample over $Z$, and $K_{Y}$ is numerically trivial over $Z$. The contraction $f$ is called 
extremal if it has relative Picard number 1 . In particular if $(Y, \Delta)$ is a Qfactorial klt pair with $\Delta$ effective and $f$ is the contraction of a $\left(K_{Y}+\Delta\right)$ negative extremal ray, it is extremal, because all the curves contracted are numerical multiples of each other, by the cone theorem [14, Theorem 3.7]. Given a $D$-flopping contraction $f: Y \rightarrow Z$, the $D$-flop is a birational morphism $f^{+}: Y^{+} \rightarrow Z$ from a normal variety $Y^{+}$such that $\operatorname{Exc}\left(f^{+}\right)$has codimension at least 2 in $Y^{+}$, and the proper transform $\left(K_{Y}+D\right)^{+}$of $\left(K_{Y}+D\right)$ on $Y^{+}$is $\mathbf{R}$-Cartier and ample over $Z$. (Sometimes we abuse terminology by referring to either the birational map $\left(f^{+}\right)^{-1} \circ f: Y \rightarrow Y^{+}$or the variety $Y^{+}$as the flop.)

Note that flops preserve the $\mathbf{Q}$-factorial property and for terminal 3-folds preserve the singularity type [14, Proposition 3.37, Theorem 6.15]. Moreover it is easy to see that if $-K_{X}$ is semiample then so is $-K_{X^{+}}$for $X^{+}$any flop of $X$, and these two line bundles have the same Iitaka dimension. We conclude that the class of 3 -folds considered in Theorem 0.1 (terminal Q-factorial Gorenstein with $-K$ semiample of positive Iitaka dimension) is preserved by flops.

Theorem 2.3. Let $X$ be a Q-factorial terminal Gorenstein 3-fold with $-K_{X}$ semiample of positive Iitaka dimension. Then the effective movable cone $\overline{M(X)}$ e decomposes as a union of effective nef cones of SQMs of X:

$$
\overline{M(X)}^{e}=\bigcup{\overline{A\left(X^{\prime}, \alpha\right)}}^{e}
$$

where the union on the right-hand side is over all $S Q M s X^{\prime} \rightarrow X$. All these $S Q M$ s are over $S$. The interiors of the cones $\overline{A\left(X^{\prime}, \alpha\right)}$ are disjoint.

Proof. Suppose $D \in \overline{M(X)}^{e}$ is an effective Q-divisor on $X$ which is not nef. By Corollary $2.2 D$ cannot be negative on a $K$-negative extremal ray, so we must have $D \cdot R<0$ for some extremal ray $R$ of $\overline{\operatorname{Curv}(X)}$ which lies in $K^{\perp}$. Choosing some $\epsilon>0$ sufficiently small, the cone theorem for the klt pair $(X, \epsilon D)$ tells us that $R$ is spanned by the class of a curve, and the contraction of $R$ exists. Note that since $R \subset K^{\perp}$, this contraction is over $S$. By $[14$, Theorem 6.14] the $D$-flop of this contraction exists, and is an SQM of $X$ over $S$. If $D$ is not a $\mathbf{Q}$-divisor, we can choose a small ample $R$-divisor $D^{\prime}$ such that $D+D^{\prime}$ is a $\mathbf{Q}$-divisor in the cone $\overline{M(X)}^{e}$ but is not nef. So as before we get a $\left(D+D^{\prime}\right)$-flopping contraction over $S$ and its $\left(D+D^{\prime}\right)$-flop over $S$. Since $D^{\prime}$ is ample, this is in particular a $D$-flop.

So given a non-nef divisor $D \in \overline{M(X)}^{e}$, there exists a $D$-flop $X \rightarrow X^{+}$. Applying this fact repeatedly (using the fact explained above that $X^{+}$satisfies the same assumptions as $X$ ), either $D$ becomes nef after a finite sequence 
of $D$-flops, or else there is an infinite sequence of $D$-flops. (Here we are abusing terminology a little: a sequence of $D$-flops really means a sequence of flops whose $(i+1)^{t h}$ member is a $D^{i}$-flop, where $D^{i}$ is the proper transform of $D$ by the composition of the first $i$ flops in the sequence.) But Kawamata [10] showed there is no infinite sequence of $D$-flops for $D$ a $\mathbf{Q}$-divisor on a Q-factorial terminal 3-fold, and as remarked in [11, Theorem 2.3] the same proof works if $D$ is an $\mathbf{R}$-divisor. We conclude that any $D \in \overline{M(X)}$ e becomes nef after a finite sequence of flops.

We have shown that any effective movable divisor belongs to one of the effective nef cones $\overline{A\left(X^{\prime}, \alpha\right)}{ }^{e}$ where $\alpha: X^{\prime} \rightarrow X$ is a sequence of flops over $S$. So we have the inclusion $\overline{M(X)}^{e} \subset \bigcup_{\alpha}{\overline{A\left(X^{\prime}, \alpha\right)}}^{e}$. The reverse inclusion is clear, since an ample divisor on any SQM $X^{\prime}$ is movable on $X$, so taking closures and intersecting with the effective cone we get $\bigcup_{\alpha}{\overline{A\left(X^{\prime}, \alpha\right)}}^{e} \subset \overline{M(X)}^{e}$.

To see that these flops give all the SQMs of $X$ up to isomorphism, suppose that $\beta: Y \rightarrow X$ is any SQM. By the argument above we have $\overline{A(Y, \beta)} \subset \bigcup_{\alpha} \overline{A\left(X^{\prime}, \alpha\right)}$, so the ample cone of $Y$ must intersect the ample cone of one of the flops, say $\alpha_{i}: X_{i} \rightarrow X$. So there exists a divisor $D$ on $X$ such that $\alpha_{i *}^{-1} D$ and $\beta_{*}^{-1} D$ are ample on $X_{i}$ and $Y$ respectively. Therefore

$$
X_{i}=\operatorname{Proj} R\left(X_{i}, \alpha_{i_{*}}^{-1} D\right) \cong \operatorname{Proj} R\left(Y, \beta_{*}^{-1} D\right)=Y
$$

and the isomorphism is compatible with $\alpha_{i}$ and $\beta$. In other words, $\left(X_{i}, \alpha_{i}\right)$ and $(Y, \beta)$ are isomorphic as SQMs of $X$.

Finally, the same argument applied to 2 SQMs $\alpha_{1}: X_{1} \rightarrow X$ and $\alpha_{2}$ : $X_{2} \rightarrow X$ shows that the interiors of the cones $\overline{A\left(X_{1}, \alpha_{1}\right)}$ and $\overline{A\left(X_{2}, \alpha_{2}\right)}$ are disjoint in $\overline{M(X)}^{e}$.

As an immediate consequence, we get Theorem 0.1 :

Corollary 2.4. Let $X$ be a Q-factorial terminal Gorenstein 3-fold, and assume that $-K_{X}$ is semiample of positive Iitaka dimension. Then the number of effective nef cones in the decomposition $\overline{M(X)}^{e}=\bigcup_{\alpha}{\overline{A\left(X^{\prime}, \alpha\right)}}^{e}$ is finite up to the action of PsAut $(X / S)$.

Proof. Under the hypotheses, Kawamata [11, Theorem 3.6, Theorem 4.4] proves that the decomposition of $\overline{M(X / S)}$ into relative nef cones is finite up to the action of PsAut $(X / S)$. In other words, there are only finitely many SQMs of $X$ over $S$ up to the action of PsAut $(X / S)$. But Theorem 2.3 shows that every SQM of $X$ is over $S$, so we get the result. 


\section{Extension of Kawamata's theorems}

In this section we adapt to our situation some results of Kawamata [11, Theorem 1.9, Theorem 2.6] concerning the local structure of the nef and movable cone. These results will be used in the next section to give a description of the relative movable cone in some cases. The proofs here are appropriately modified versions of Kawamata's, though we give more details.

Theorem 3.1. Let $(X / Z, \Delta)$ be a klt Calabi-Yau pair (of any dimension). Then the cone

$$
\overline{A(X / Z)} \cap B(X / Z)=\overline{A(X / Z)}^{e} \cap B(X / Z)
$$

is locally rational polyhedral inside the big cone $B(X / Z)$. Moreover, any face $F$ of this cone corresponds to a birational contraction $\phi: X \rightarrow Y$ over $Z$ by the equality $F=\phi^{*}(\overline{A(Y / Z)} \cap B(Y / Z))$.

We will only need the theorem in the case $Z=\operatorname{Spec} k$, but since the proof of the more general statement is identical, it makes sense to include it here.

Proof. The proof of the first statement works just as Kawamata's proof for Calabi-Yau fibre spaces [9, Theorem 5.7], replacing $K_{X}$ by $K_{X}+\Delta$ wherever necessary. Here is the argument in more detail. Let $(X / Z, \Delta)$ be a klt Calabi-Yau pair, and $D$ a $\mathbf{Q}$-divisor which is effective but not nef over $Z$. For a sufficiently small positive number $\epsilon$, the pair $(X, \Delta+\epsilon D)$ is again klt, so by the relative cone theorem we have

$$
\overline{\operatorname{Curv}(X / Z)}=\overline{\operatorname{Curv}(X / Z)} \cap\left\{K_{X}+\Delta+\epsilon D \geq 0\right\}+\Sigma_{j} R_{j}
$$

where the $R_{j}$ are extremal rays which are negative with respect to $K_{X}+$ $\Delta+\epsilon D \equiv_{Z} \epsilon D$. Now suppose that $D$ is big over $Z$ : then we can write $D \equiv_{Z}$ $D_{1}+D_{2}$, where $D_{1}$ is effective over $Z$ and $D_{2}$ is ample over $Z$. Then the relative cone theorem for the klt pair $\left(X, \Delta+\epsilon D_{1}\right)$ says that the number of extremal rays $R_{j}^{\prime}$ with $\left(\epsilon D_{1}+\epsilon D_{2}\right) \cdot R<0$ is finite, because $\epsilon D_{2}$ is ample over $Z$. But now $\left(\epsilon D_{1}+\epsilon D_{2}\right) \cdot R<0$ implies that $D_{1} \cdot R<0$, so the rays $R_{j}$ appearing in the displayed equation above are a subset of the set of rays $R_{j}^{\prime}$. So there are finitely many extremal rays of $\overline{\operatorname{Curv}(X / Z)}$ which are negative with respect to $D$.

The nef cone of $X$ over $Z$ is the intersection $\bigcap_{R}\left\{x \in N^{1}(X / Z) \mid x \cdot R \geq\right.$ $0\}$ of the nonnegative half-spaces in $N^{1}(X / Z)$ for all extremal rays $R$ of 
$\overline{\operatorname{Curv}(X / Z)}$. By the previous paragraph, $D$ lies in all but finitely many of these half-spaces. This means that the part of the boundary of $\overline{A(X / Z)}$ visible from $D$ is defined by a finite set of rational hyperplanes. Since this is true for an arbitrary non-nef rational point $D$ in the big cone, we conclude that $\overline{A(X / Z)}$ is locally rational polyhedral inside the nef cone.

For the second statement, it suffices to show that a nef and big divisor over $Z$ is semiample over $Z$. This will follow from the relative version of the Basepoint-free theorem [14, Theorem 3.3] if we show that $D$ being nef and big over $Z$ implies that $D-K_{X}-\Delta$ is nef and big over $Z$. But by definition of a Calabi-Yau pair $K_{X}+\Delta=0$ in $N^{1}(X / Z)$, and the property of being nef and big is defined on the level of numerical classes [14, Proposition 2.61], so we are done.

Theorem 3.2. Let $X$ be a $\mathbf{Q}$-factorial terminal Gorenstein 3-fold with $-K_{X}$ semiample of positive Iitaka dimension. Then the decomposition

$$
\overline{M(X)}^{e} \cap B(X)=\bigcup{\overline{A\left(X^{\prime}, \alpha\right)}}^{e} \cap B(X)
$$

is locally finite inside the big cone $B(X)$ in the following sense: if $\Sigma$ is a closed convex cone contained in $B(X) \cup\{0\}$, then only a finite number of the cones ${\overline{A\left(X^{\prime}, \alpha\right)}}^{e} \cap B(X)$ intersect $\Sigma$.

Proof. First recall from Section 1 that under the stated hypotheses, there exists a Q-divisor $\Delta$ on $X$ such that $(X, \Delta)$ is a klt Calabi-Yau pair. So Theorem 3.1 tells us that each nef cone in the decomposition is locally rational polyhedral inside the big cone $B(X)$.

Now let $x \in \overline{M(X)}^{e} \cap B(X)$. By Theorem 2.3 there exists an SQM $\left(X_{0}\right.$, $\left.\alpha_{0}\right)$ of $X$ such that $x \in \overline{A\left(X_{0}, \alpha_{0}\right)}{ }^{e}$. Let $F$ be the face of $\overline{A\left(X_{0}, \alpha_{0}\right)}$ whose interior contains $x$. I make two claims at this point. The first claim is that the number of effective nef cones ${\overline{A\left(X_{i}, \alpha_{i}\right)}}^{e}$ which contain $F$ as a face is finite. The second claim is that one can choose a small open cone $\Sigma_{x}$ containing $x$ such that $\overline{M(X)}{ }^{e} \cap \Sigma_{x}$ intersects only finitely many effective nef cones $\overline{A\left(X_{i}, \alpha_{i}\right)}{ }^{e}$. Given these two claims the result then follows: if $\Sigma$ is a closed convex cone as in the statement of the theorem, the cones $\left\{\overline{M(X)} e^{e} \cap \Sigma_{x} \cap\right.$ $\Sigma \mid x \in \Sigma\}$ give an open cover of $\overline{M(X)}^{e} \cap \Sigma$. Furthermore, since $B(X)$ is contained in the effective cone and $\Sigma$ is contained in $B(X)$, we have the following equalities of cones:

$$
\overline{M(X)}^{e} \cap \Sigma=\overline{M(X)}^{e} \cap B(X) \cap \Sigma=\overline{M(X)} \cap B(X) \cap \Sigma=\overline{M(X)} \cap \Sigma .
$$


In particular $\overline{M(X)}^{e} \cap \Sigma$ is a closed cone in $N^{1}(X)$, so its projectivisation is compact. Therefore the open cover we mentioned has a finite subcover. Since each $\Sigma_{x}$ intersects only finitely many nef cones, this gives the result.

It remains to prove the two claims. For the first, suppose $F$ is a face of some nef cone $\overline{A\left(X_{0}, \alpha_{0}\right)}$. Then we have by Theorem $3.1 \mathrm{a}$ birational morphism $f_{0}: X_{0} \rightarrow Y$ such that $F=f_{0}^{*} \overline{A(Y)}$.

I claim that the number of nef cones ${\overline{A\left(X_{i} / Y\right)}}^{e}$ for the SQMs of $X_{0}$ over $Y$ is finite. To see this, choose an $f_{0}$-ample Cartier divisor $D$ on $X_{0}$ : by [14, Lemma 6.28] there is an effective divisor $E$ such that $-D$ is $f_{0}$-linearly equivalent to $E$. Also as explained before, since $-K_{X}$ is semiample we can find an effective divisor $\Delta$ such that $\left(X_{0}, \Delta\right)$ is a klt Calabi-Yau pair. So by the relative cone theorem for the klt pair $\left(X_{0}, \Delta+\epsilon E\right)$ (where $\epsilon$ is a sufficiently small positive number) the relative cone of curves $\overline{\operatorname{Curv}\left(X_{0} / Y\right)}$ is rational polyhedral.

Now suppose there were infinitely many nef cones ${\overline{A\left(X_{i} / Y\right)}}^{e}$. Since each $X_{i}$ is $\mathbf{Q}$-factorial and terminal, and for each $i$ there is a divisor $\Delta_{i}$ such that $K_{X_{i}}+\Delta_{i} \equiv 0$, we can apply Kawamata's decomposition theorem [14, Theorem 6.38] to conclude that the birational map $X_{i} \rightarrow X_{j}$ (given by the marking) between any two SQMs decomposes as a finite sequence of flops over $Y$. (More precisely, we are extending the result of Kawamata from the case where $K_{X_{i}}$ is nef over $Y$ to the case where $K_{X_{i}}+\Delta_{i}$ is nef over $Y$. But examining the proof we see as before that it depends on applying the cone theorem for a klt pair $(X, \epsilon D)$, and everything works just as well with the klt pair $(X, \Delta+\epsilon D)$.)

Now fix a model $f_{0}: X_{0} \rightarrow Y$ and an ample effective divisor $D_{0}$ on $X_{0}$. As in the previous paragraph, there is an effective divisor $E_{0}$ on $X_{0}$ which is $f_{0}$-linearly equivalent to $-D_{0}$. Then any other $X_{i}$ is obtained from $X_{0}$ by a finite sequence of $E_{0}$-flops. Since each cone $\overline{\operatorname{Curv}\left(X_{i} / Y\right)}$ is rational polyhedral, there are only finitely many ways to do a flop at each stage. But we assumed that there are infinitely many nef cones, so by König's Lemma, there must be an infinite sequence of $E_{0}$-flops. This contradicts the fact mentioned in the proof of Theorem 2.3 that any sequence of terminal 3-flops is finite. So there can be only finitely many nef cones $\overline{A\left(X_{i} / Y\right)}{ }^{e}$. Call the corresponding SQMs $X_{1}, \ldots, X_{n}$.

Finally suppose that $X_{\alpha}$ is any SQM of $X$ such that ${\overline{A\left(X_{\alpha}\right)}}^{e}$ contains $F$ as a face. As before, we get a birational contraction $f_{\alpha}: X_{\alpha} \rightarrow Y$. By the previous paragraph, we must have ${\overline{A\left(X_{\alpha} / Y\right)}}^{e}={\overline{A\left(X_{i} / Y\right)}}^{e}$ for some $i \in$ $\{1, \ldots, n\}$. This means $X_{\alpha}$ is isomorphic to $X_{i}$ as an SQM over $Y$, so in particular isomorphic to $X_{i}$ as an SQM of $X$ (over Spec $k$ ). In other words 
${\overline{A\left(X_{\alpha}\right)}}^{e}={\overline{A\left(X_{i}\right)}}^{e}$ as cones in $N^{1}(X)$, which completes the proof of the first claim.

Now for the second claim: namely, given $x \in \overline{M(X)}^{e} \cap B(X)$ one can choose a small open cone $\Sigma_{x}$ containing $x$ such that $\overline{M(X)}^{e} \cap \Sigma_{x}$ intersects only finitely many effective nef cones $\overline{A\left(X_{i}\right)^{e}}$. Recall that $x$ belongs to the interior of a face $F$ of some nef cone, and we have just shown that there are only finitely many nef cones which contain $F$ as a face. Call these nef cones $\overline{A\left(X_{i}\right)}$, for $i=1, \ldots, k$. By Theorem 3.1 each nef cone is locally rational polyhedral near $x$, so for any sufficiently small open cone $K$ containing $x$, the only faces of these nef cones which meet $K$ are the faces which contain $F$ as a face.

If the claim is not true, then any open neighbourhood of $x$ inside $\overline{M(X)}$ must meet infinitely many nef cones, and in particular must meet infinitely many nef cones which do not contain $F$ as a face (because there are only finitely many nef cones which do contain $F$ as a face). Let us assume this is the case, and derive a contradiction.

Let $K$ be an open cone around $x$ chosen small enough (as explained above) so that the only faces of the nef cones $\overline{A\left(X_{i}\right)}$ (for $\left.i=1, \ldots, k\right)$ which meet $K$ are those which contain $F$ as a face. I claim that the union $U=$ $\overline{A\left(X_{1}\right)} \cup \cdots \cup \overline{A\left(X_{k}\right)}$ must have an 'exposed face' inside $\overline{M(X)} \cap K$ : that is, there is a face $F^{\prime}$ of one of these nef cones which lies on the relative boundary inside $\overline{M(X)} \cap K$ of the set $U$. To see this, note that if no such exposed face exists, then every point of $U \cap K$ would be in the relative interior of $U \cap K$ inside $\overline{M(X)} \cap K$, implying that $U \cap K$ is an open subset of $\overline{M(X)} \cap K$. On the other hand, $U \cap K$ is a finite union of closed sets in $\overline{M(X)} \cap K$, so is closed. Therefore $U \cap K$ must be the whole of $\overline{M(X)} \cap K$. So $\overline{M(X)} \cap K$ is an open neighbourhood of $x$ inside $\overline{M(X)}$ which meets only finitely many nef cones, contradicting the hypothesis of the previous paragraph. This contradiction implies that $U$ must have an exposed face inside $\overline{M(X)} \cap K$.

Since the relative boundary of $U$ inside $\overline{M(X)} \cap K$ is itself a polyhedral complex, there must in fact be an exposed face of codimension 1 inside $\overline{M(X)} \cap K$. Choose such a face $F^{\prime}$; by our choice of the cone $K$ in the previous paragraph we know that $F^{\prime}$ contains $F$ as a face. Assume that $F^{\prime}$ is a face of a nef cone $\overline{A\left(X_{1}\right)}$. Then $F^{\prime}$ is dual to an extremal ray $R$ of the closed cone of curves $\overline{\operatorname{Curv}\left(X_{1}\right)}$. Choose an interior point $x^{\prime}$ of $F^{\prime}$ : since $F^{\prime}$ is exposed, any open neighbourhood of $x^{\prime}$ inside $\overline{M(X)} \cap K$ contains points which do not belong to the set $U$. Choose a point $x^{\prime \prime}$ in the complement of $U$ with the property that $x^{\prime \prime}$ is negative on the extremal ray $R$, but is positive 
on all other extremal rays of $\overline{\operatorname{Curv}\left(X_{1}\right)}$. (Here we are using the conclusion of Theorem 3.1 that $\overline{A\left(X_{1}\right)}$ is locally rational polyhedral inside the big cone, so in a neighbourhood of $x^{\prime}$ it is defined by finitely many hyperplanes. By the standard argument with the cone theorem (applied to a klt pair $\left(X_{1}, \epsilon x^{\prime \prime}\right)$ for $\epsilon$ sufficiently small), we obtain the contraction of the extremal ray $R$, and the corresponding flop. Call the flopped variety $X^{\prime}$ : then $\overline{A\left(X^{\prime}\right)}$ intersects $\overline{A\left(X_{1}\right)}$ along the face $F^{\prime}$, so in particular contains $F$ as a face. In other words, $\overline{A\left(X^{\prime}\right)}$ must equal $\overline{A\left(X_{i}\right)}$ for some $i=1, \ldots, k$.

On the other hand since the two nef cones $\overline{A\left(X^{\prime}\right)}$ and $\overline{A\left(X_{i}\right)}$ meet along the codimension-1 face $F^{\prime}$, the union $\overline{A\left(X_{1}\right)} \cup \overline{A\left(X^{\prime}\right)}$, which we now know is a subset of $U$, contains an open neighbourhood of every interior point of $F^{\prime}$. This contradicts the fact that $F^{\prime}$ is an exposed face of $U$.

The contradiction shows that our original assumption that every open neighbourhood of $x$ inside $\overline{M(X)}$ meets infinitely many nef cones is untenable, and so the second claim is proven.

\section{Relative movable cone and lifting}

In this section we describe the relative movable cone completely in the case that $-K_{X}$ has Iitaka dimension 2 and the fibration $f: X \rightarrow S$ has only 1dimensional fibres. Roughly speaking, we show that the relative movable cone is 'as simple as possible': it is bounded by the numerical classes of moving families of fibre components. The precise statement is given in Theorem 4.6. This description allows us to prove a finiteness result for the (absolute) movable cone: there is a rational polyhedral cone which intersects only finitely many nef cones, and whose translates cover the whole movable cone (Theorem 4.10). As an application, we get a proof of the full movable cone conjecture in a special case (Example 4.12). Throughout this section we work under the following assumption:

Assumption 1: $X$ is a $\mathbf{Q}$-factorial terminal Gorenstein 3-fold, the anticanonical bundle $-K_{X}$ is semiample of Iitaka dimension 2, and the fibres of the contraction morphism $f: X \rightarrow S$ defined by a suitable multiple of $-K_{X}$ are all 1-dimensional.

It is not clear to me to what extent the methods of this section can be adapted to study the non-equidimensional case, or the case when $-K_{X}$ has Iitaka dimension 1. In any case, the conditions of Assumption 1 still permit at least one nontrivial class of examples, discussed in Example 4.12. 
We begin by introducing some notation and terminology. We denote the generic fibre of the morphism $f: X \rightarrow S$ by $X_{\eta}$. Since $f$ is given by some sections of some power of $-K_{X}$, adjunction tells us that $X_{\eta}$ is a smooth curve of genus 1 , and so we refer to $f$ as an elliptic fibration. (Note that we do not assume the existence of a rational section of $f$.) The MordellWeil group of $f$ is the abelian group $\operatorname{Pic}^{0}\left(X_{\eta}\right)$ of degree- 0 line bundles on $X_{\eta}$. The translation action of the Mordell-Weil group on $X_{\eta}$ extends to an action on $X$ by pseudo-automorphisms over $S$, so we have an inclusion $\operatorname{Pic}^{0}\left(X_{\eta}\right) \subseteq \operatorname{PsAut}(X / S)$. To prove any form of the movable cone conjecture, it is therefore sufficient to prove the corresponding statement with $\operatorname{Pic}^{0}\left(X_{\eta}\right)$ in place of $\operatorname{PsAut}(X / S)$, and this is what Kawamata does in his proof for Calabi-Yau fibre spaces.

We start by quoting the following lemma [11, Lemma 3.1] which allows us to pull back arbitrary Weil divisors from $S$ to $X$.

Lemma 4.1. Let $f: X \rightarrow S$ be as in Assumption 1. Then $S$ is $\mathbf{Q}$-factorial.

Next we define the subspace $T(X / S)$ of relatively trivial divisors on $X$ to be the kernel of the natural projection $N^{1}(X) \rightarrow N^{1}(X / S)$. In other words $T(X / S)$ is the subspace of classes which have degree zero on all curves in $X$ which map to a point in $S$. It is clear that $T(X / S)$ contains $f^{*}\left(N^{1}(S)\right)$ as a subspace, but in general they need not be equal [14, Proposition 3.27]. The first thing to note is that $T(X / S)$ does not depend on the model of $X$ we choose:

Lemma 4.2. Suppose $X^{\prime} \rightarrow S$ is any $S Q M$ of $X$ (automatically over $S$ by Theorem 2.3). Then $T(X / S)=T\left(X^{\prime} / S\right)$ as subspaces of $N^{1}(X)=N^{1}\left(X^{\prime}\right)$.

Proof. By Theorem $2.3 X^{\prime}$ is obtained from $X$ by flopping fibral curves. Suppose first $X \rightarrow X^{\prime}$ is the flop of a single curve $C$ with numerical class $[C]$. The canonical identification $N^{1}(X) \cong N^{1}\left(X^{\prime}\right)$ gives a dual identification $N_{1}(X) \cong N_{1}\left(X^{\prime}\right)$ which takes $[C]$ to $-[C]$ and, for any other curve $\Gamma$ in $X$, takes $[\Gamma]$ to $[\Gamma]+n[C]$ for some integer $n$ (depending on $\Gamma$ ). Therefore the identification takes the subspace $N_{1}(X / S)$ to $N_{1}\left(X^{\prime} / S\right)$.

If $X \rightarrow X^{\prime}$ is any sequence of flops, applying the same argument repeatedly shows that $N_{1}(X / S)$ is identified with $N_{1}\left(X^{\prime} / S\right)$. Now $T(X / S)=$ $N_{1}(X / S)^{\perp} \subset N^{1}(X)$, giving the result.

Next, a prime divisor $D$ on $X$ is defined to be vertical if $f(D) \neq S$. We denote by $V(X / S)$ the subspace of $N^{1}(X / S)$ spanned by vertical divisors, and denote by $v$ the dimension of $V(X / S)$. A prime divisor $D$ is exceptional 
over $S$ if there is a SQM $f^{\prime}: X^{\prime} \rightarrow S$ of $X$ over $S$ and a divisorial contraction $\phi: X^{\prime} \rightarrow Y$ over $S$ with exceptional divisor $E$ such that $D$ is the proper transform of $E$. We will need the following facts about vertical and exceptional divisors [11, Lemma 3.1, Lemma 3.2]:

Lemma 4.3. Let $f: X \rightarrow S$ be as in Assumption 1 .

1. If $D$ is an vertical prime divisor, and $D$ does not contain the fibre of the restriction $f^{-1} f(D) \rightarrow D$ over the generic point of the curve $f(D)$, then $D$ is exceptional over $S$.

2. The vector space $V(X / S)$ of vertical divisor classes is spanned by the classes of exceptional divisors over $S$.

Since we assume that all fibres of $f: X \rightarrow S$ are 1-dimensional, all fibres have the same numerical class in $N_{1}(X)$, which we denote by $F$. Now suppose $C$ is an irreducible curve on $S$. The preimage $f^{-1}(C)$ of this curve is (as a set) a union $D_{1} \cup D_{2} \cup \cdots \cup D_{k}$ of vertical prime divisors on $X$. The settheoretic intersection $D_{i} \cap f^{-1}(p)$ of the divisor $D_{i}$ with a fibre of $f$ over the generic point of $C$ is a curve whose numerical class we denote by $F_{i}$. Since the numerical class of any fibre is equal to $F$, we get an expression $m_{1} F_{1}+\cdots+m_{k} F_{k}=F$, where the $m_{i}$ are positive integers. This implies that $D_{i} \cdot\left(m_{1} F_{1}+\cdots+m_{k} F_{k}\right)=0$ for each $i$, because $D_{i}$ is vertical. On the other hand since the fibres of $f$ are connected, if $k \geq 2$ (which by Lemma 4.3 means that the $D_{i}$ are exceptional over $S$ ) we must have $D_{i} \cdot F_{j}>0$ for some $j \neq i$, and therefore $D_{i} \cdot F_{i}<0$. This shows in particular that the classes $F_{i}$ for $i=1, \ldots, k$ are all distinct in $N_{1}(X)$. Furthermore if $D_{1}$ and $D_{2}$ are exceptional prime divisors over $S$ such that $f\left(D_{1}\right) \neq f\left(D_{2}\right)$, with the corresponding curves having classes $F_{1}$ and $F_{2}$ then we have $D_{1} \cdot F_{2}=D_{2}$. $F_{1}=0$, again implying that $F_{1}$ and $F_{2}$ are distinct classes. We conclude that for each exceptional prime divisor $D_{i}$ over $S$ we have a class $F_{i} \in N_{1}(X)$ of a fibral curve of $f$, and distinct divisors give distinct classes. Since $f$ has 1-dimensional fibres, there can be only finitely many such classes (because there are only finitely many decompositions of $F$ in the monoid $\left.\operatorname{Curv}(X)_{\mathbf{z}}\right)$, so there are only finitely many exceptional divisors over $S$. From now on $D_{1}, \ldots, D_{n}$ will denote these exceptional divisors over $S$, and $F_{1}, \ldots, F_{n}$ the corresponding classes of fibral curves of $f$.

One other piece of notation will be useful for us. We partition the set $\mathcal{D}=\left\{D_{1}, \ldots, D_{n}\right\}$ of exceptional divisors over $S$ into subsets $\mathcal{D}_{1}, \ldots, \mathcal{D}_{r}$ by saying that $D_{i}$ and $D_{j}$ belong to the same member of the partition if the curves $f\left(D_{i}\right)$ and $f\left(D_{j}\right)$ are the same. Denote by $C_{p}$ the curve in $S$ which is the common image of the divisors belonging to the member $\mathcal{D}_{p}$ of the 
partition. Note that $f^{*}\left(C_{p}\right)=\sum_{\left\{i \mid D_{i} \in \mathcal{D}_{p}\right\}} \mu_{i} D_{i}$ for some positive rational numbers $\mu_{i}$, so in particular $\sum_{\left\{i \mid D_{i} \in \mathcal{D}_{p}\right\}} \mu_{i} D_{i}=0$ in $N^{1}(X / S)$. Also, by the previous paragraph, for each $\mathcal{D}_{p}$ there are positive integers $m_{i}$ such that $\sum_{\left\{i \mid D_{i} \in \mathcal{D}_{p}\right\}} m_{i} F_{i}=F$, the class of a fibre.

Now we can begin to describe the relative movable cone of $X$ over $S$. We need the following 'negativity lemma' for sums of exceptional divisors over $S$.

Lemma 4.4. Suppose $D_{i}$ and $F_{j}$ are as defined above. Then a class $x=$ $\sum_{i} r_{i} D_{i} \in N^{1}(X)$ satisfies $x \cdot F_{j} \geq 0$ for all $j=1, \ldots, n$ if and only if $x=$ $f^{*}(C)$ for some $\mathbf{R}$-divisor $C$ on $S$, in which case $x \cdot F_{j}=0$ for all $j . A$ class $y=\sum_{i} s_{i} F_{i} \in N_{1}(X)$ satisfies $D_{j} \cdot y \geq 0$ for all $j=1, \ldots, n$ if and only if $y=s F$ for some real number $s$, in which case $D_{j} \cdot y=0$ for all $j$.

Proof. One direction is trivial: if $x$ is the pullback of an $\mathbf{R}$-divisor on $S$ the intersection numbers $x \cdot F_{j}$ are obviously 0 . Conversely assume $x=\sum_{i} r_{i} D_{i}$ satisfies $x \cdot F_{j} \geq 0$ for all $j=1, \ldots, n$. We want to show that $x$ is the pullback of an $\mathbf{R}$-divisor on $S$.

First, for each $\mathcal{D}_{p}$ in the partition defined above there are positive integers $m_{i}$ such that $\sum_{\left\{i \mid D_{i} \in \mathcal{D}_{p}\right\}} m_{i} F_{i}=F$. Therefore since $D_{i} \cdot F=0$ for all $D_{i}$, the condition $x \cdot F_{j} \geq 0$ for all $j$ is equivalent to the condition $x \cdot F_{j}=0$ for all $j$.

Next, the partition we defined gives a decomposition of $x$ as $x=x_{1}+$ $\cdots+x_{r}$, where $x_{p}=\sum_{\left\{i \mid D_{i} \in \mathcal{D}_{p}\right\}} r_{i} D_{i}$. Note that $x_{p} \cdot F_{j}=0$ whenever $j$ is not in $\mathcal{D}_{p}$, so the condition $x \cdot F_{j}=0$ for each $j$ is equivalent to the following: for each $j$, we have $x_{p} \cdot F_{j}=0$ for the unique $p$ such that $D_{j} \in \mathcal{D}_{p}$. So we can reduce to the case where $x=x_{p}$ for some $p$ : that is, $x$ is a real linear combination of divisors $D_{i}$ all belonging to some member $\mathcal{D}_{p}$ of the partition, that is all of whose images $f\left(D_{i}\right) \subset S$ are the same curve. Call this curve $C$, and write $f^{*}(C)=\sum_{\left\{i \mid D_{i} \in \mathcal{D}_{p}\right\}} \mu_{i} D_{i}$. We want to show that there exists a real number $\lambda$ such that $r_{i}=\lambda \mu_{i}$ for all $i$, implying $x=\lambda f^{*}(C)$.

To see this, choose a general curve $\Gamma \subset S$ intersecting $C$ transversely in $m>0$ points. We will restrict to the surface $\Sigma=f^{-1}(\Gamma)$. The restriction of $D_{i}$ to $\Sigma$ is a union of $m$ disjoint curves each with class $F_{i}$, so we get $x_{\mid \Sigma}=m\left(\sum_{i} r_{i} F_{i}\right)$. Since $x \cdot F_{j}=0$ for each $j$, we get $\left(x_{\mid \Sigma}\right)^{2}=0$. But the Hodge index theorem on $\Sigma$ then implies that $x_{\mid \Sigma}$ is a multiple of the class $\Phi$ of a fibre of $\Sigma \rightarrow \Gamma$ : say $x_{\mid \Sigma}=\lambda \Phi$. By looking at the fibre over a point of $C$ we see that $\Phi=\sum_{i} \mu_{i} F_{i}$, so we conclude that $\sum_{i} r_{i} F_{i}=\lambda\left(\sum_{i} \mu_{i} F_{i}\right)$, hence $x=\lambda f^{*}(C)$, as required.

The proof of the claim about the classes $y=\sum_{i} s_{i} F_{i}$ is analogous. 
Corollary 4.5. The dual vector space

$$
F(X / S)=N_{1}(X / S) /\left\{x \in N_{1}(X / S) \mid D \cdot x=0 \text { for all } D \in V(X / S)\right\}
$$

of the space $V(X / S)$ of vertical divisors is spanned by the classes $F_{1}, \ldots, F_{n}$.

Theorem 4.6. Let $D_{i}$ and $F_{j}$ be as defined above. Then the relative effective cone and the relative movable cone of $X$ over $S$ are as follows:

$$
\begin{aligned}
B^{e}(X / S) & =\left\{x \in N^{1}(X / S): x \cdot F>0\right\} \cup \mathbf{R}_{+}\left\{\left[D_{i}\right]\right\} \cup\{0\} \\
\overline{M(X / S)}^{e} & =\left\{x \in N^{1}(X / S): x \cdot F>0, x \cdot F_{i} \geq 0(i=1, \ldots, n)\right\} \cup\{0\} .
\end{aligned}
$$

Proof. First suppose that $D$ is an effective class over $S$ with degree $k \leq 0$ on the generic fibre. There exists a nonempty open set $U \subset S$ such that $D\left(f^{-1}(U)\right) \neq 0$. Choose a nonzero section $s \in D\left(f^{-1}(U)\right)$. Then the class of the divisor $\Delta=\overline{\{s=0\}}$ differs from $D$ only on the codimension-1 subset $X \backslash f^{-1}(U)$ : that is, there is an effective divisor $D^{\prime}$ supported on $X \backslash f^{-1}(U)$ such that $\Delta=D+D^{\prime}$ in $N^{1}(X)$. In particular $D$ and $\Delta$ have the same degree $k$ on the generic fibre. Since $\Delta$ is effective this implies $k=0$. Moreover $k=0$ implies that $\Delta$ is a sum of vertical (prime) divisors so by Lemma 4.3 its class belongs to the cone $V$ spanned by $f^{*}\left(B^{e}(S)\right)$ and the $D_{i}$. Finally the divisor $\Delta-D$ is supported in $X \backslash f^{-1}(U)$ therefore its support maps onto a curve in $S$. So any divisor in the support of $\Delta-D$ must also have class in the cone $V$. So for any effective class $D$ over $S$ with degree $\leq 0$ on the generic fibre we can write $D=V_{1}-V_{2}$ where $V_{i}$ are classes in $V$. The image of $V$ in $N^{1}(X / S)$ is the cone $\mathbf{R}_{+}\left\{\left[D_{i}\right]\right\}$, which is closed under negation since for any $i$, there is member $\mathcal{D}_{p}$ of the partition which contains $D_{i}$, and there exist positive rational numbers $m_{k}$ such that $\sum_{k: D_{k} \in \mathcal{D}_{p}} m_{k} D_{k}=0$ in $N^{1}(X / S)$. Therefore $[D]=\left[V_{1}\right]-\left[V_{2}\right]$ belongs to this cone as claimed. This proves that the left-hand side of the first equation is contained in the right-hand side. To prove the reverse inclusion, first note that if a divisor $D$ has positive degree on an irreducible fibre $F$ then the restriction $D_{\mid F}$ is ample hence effective. But standard results on semicontinuity of cohomology [8, Corollary III.12.9] show that for any positive integer $m$, any section of $m D_{\mid F}$ is the restriction of a section in $m D\left(f^{-1}(U)\right)$ for $U \subset S$ some open subset. Therefore by definition $m D$ and hence $D$ is effective over $S$. Finally, all divisors in the cone $V$ are effective by definition hence effective over $S$, so all elements of $\mathbf{R}_{+}\left\{\left[D_{i}\right]\right\}$ lie in the relative effective cone. This completes the proof of the claim about the relative effective cone.

Now we must prove the claim about the relative movable cone. First note that if $D$ is a class in $N^{1}(X)$ with $D \cdot F_{i}<0$ for some $i$, then $D$ 
cannot be movable over $S$. For suppose $C$ is a curve in $X$ with class $F_{i}$. If there was an open set $U \subset S$ containing the point $f(C)$ and a section of $D\left(f^{-1}(U)\right)$ not vanishing identically along $C$ we would have $D \cdot C \geq 0$ contradicting our assumption: therefore every such curve $C$ is contained in Supp Coker $\left(f^{*} f_{*} \mathcal{O}_{X}(D) \rightarrow \mathcal{O}_{X}(D)\right)$. Since these curves $C$ fill up an open set in the divisor $D_{i}$ we conclude that $D$ cannot be movable over $S$. So the relative movable cone is contained in the cone $\left\{x \cdot F_{i} \geq 0\right.$ for all $\left.i\right\}$. If moreover $x$ is a nonzero class effective and movable over $S$, it must have $x \cdot F>0$. For otherwise by the description of $B^{e}(X / S)$ we would have $x \in \mathbf{R}_{+}\left\{\left[D_{i}\right]\right\}$. Any nonzero point in this cone can be written in the form $x=\sum r_{i}\left[D_{i}\right]$; by Lemma 4.4, since $x$ is not pulled back from $S$, this implies $x \cdot F_{i}<0$ for some $i$. So we have shown that left-hand side is contained in the right-hand side in the second equality above.

Conversely suppose that $x \in N^{1}(X / S)$ satisfies $x \cdot F_{i} \geq 0$ for all $i$ and $x \cdot F>0$ : we want to show that $x$ belongs to the relative effective movable cone. First note that any such $x$ is effective over $S$ by our description of the relative effective cone. Next suppose that $D$ is a divisor class with $D \cdot F_{i}>0$ for each $i$. Since the class of a fibre $F$ is a sum of classes $F_{i}$, the restriction of such a $D$ to any irreducible fibre is ample. Also since $D \cdot F_{i}>0$ for each $i$ the restriction of $D$ to each component of the fibre over the generic point of each curve $f\left(D_{i}\right)$ is ample. Again by the semicontinuity result mentioned above, for any integer $m>0$ any section of the line bundle $m D_{\mid F}$ comes from a section in $m D\left(f^{-1}(U)\right)$ for some open $U \subset S$ containing $f(F)$. Therefore if we choose an integer $m$ sufficiently large so that $m D_{\mid} F$ is very ample for some fibral curve $F$, then Supp $\operatorname{Coker}\left(f^{*} f_{*} \mathcal{O}_{X}(m D) \rightarrow \mathcal{O}_{X}(m D)\right)$ does not contain any point in the curve $F$. We conclude that the relative stable base locus $B s_{\mathbf{Q}}(D, f):=\cap_{m \geq 1} \operatorname{Supp} \operatorname{Coker}\left(f^{*} f_{*} \mathcal{O}_{X}(m D) \rightarrow \mathcal{O}_{X}(m D)\right)$ does not contain any point in any irreducible fibre or any point of the fibre over the generic point of one of the curves $f\left(D_{i}\right)$.

Suppose that the set $B s_{\mathbf{Q}}(D, f)$ contains a prime divisor $\Delta$. We have just seen that $\Delta$ must be disjoint from all irreducible fibres of $f$, so it must be vertical. Also $\Delta$ is disjoint from all components of the fibre of $f$ over the generic point of $f\left(D_{i}\right)$, so it is not one of the $D_{i}$. Since the fibre of $f$ over every point of $f(\Delta)$ is reducible, I claim that $\Delta \neq f^{-1} f(\Delta)$, as sets. To prove this, suppose equality holds: then $\Delta$ would contain the fibre of $f$ over every point of $f(\Delta)$. Suppose the fibre of $f$ over the generic point of $f(\Delta)$ is a union $C_{1} \cup \cdots \cup C_{r}$ of irreducible curves (with $r>1$ ). Taking the closure of the curve $C_{i}$ gives a divisor $\Delta_{i}$ properly contained in $\Delta$. But by assumption $\Delta$ is prime, a contradiction. So by Kawamata [11, Lemma 3.1] the divisor $\Delta$ must be exceptional over $S$, therefore equal to one of the $D_{i}$. 
This is again a contradiction. We conclude that no such divisor $\Delta$ can exist, so $B s_{\mathbf{Q}}(D, f)$ contains no divisors. By the relative version of 'stabilisation of the base locus' [15, Proposition 2.1.21] $D$ is therefore movable over $S$.

We have shown that any Cartier divisor class $[D]$ with $[D] \cdot F>0$ and $[D] \cdot F_{i}>0$ for all $i$ belongs to the relative movable cone, so the same is true for rational classes. To complete the proof we observe that any point in the cone $\left\{x \in N^{1}(X / S): x \cdot F>0, x \cdot F_{i} \geq 0\right.$ for all $\left.i\right\}$ is the limit of rational classes $x_{\alpha}$ with $x_{\alpha} \cdot F_{i}>0$ for all $i$. We have just proved that each class $x_{\alpha}$ belongs to the closed cone $\overline{M(X / S)}$ and therefore so does their limit $x$.

Lemma 4.7. The projection $\overline{M(X)}^{e} \rightarrow \overline{M(X / S)}^{e}$ is surjective.

Proof. By Theorem 4.6 we know that $\overline{M(X / S)}^{e}$ is generated as a convex cone by the classes of Cartier divisors, so it is enough to prove that if $D$ is a nonzero Cartier divisor whose class in $N^{1}(X / S)$ lies in $\overline{M(X / S)}{ }^{e}$, then $m D+\sum_{k} \nu_{k} f^{*}\left(A_{k}\right)$ is effective and movable for some integers $m>0$ and $\nu_{k}$ and some ample divisors $A_{k}$ on $S$.

By our description of $\overline{M(X / S)}^{e}$ we see that any nonzero class in that cone is big over $S$; also, by Theorem 2.3 we know that any class in that cone belongs to ${\overline{A\left(X^{\prime} / S\right)}}^{e}$ for some SQM $X^{\prime}$ of $X$. The statement of the lemma is unaffected if we replace $X$ by some SQM, so we can assume without loss of generality that $D$ is nef over $S$ and big over $S$. By the relative BasepointFree theorem, $D$ is then semiample over $S$ : that is, for some positive integer $m$ the morphism $f^{*} f_{*} \mathcal{O}(m D) \rightarrow \mathcal{O}(m D)$ is a surjection. In particular, we can choose an open set $U \subset S$ such that the restriction of $m D$ to $f^{-1}(U)$ is basepoint-free. Therefore we can choose 2 sections $s_{1}, s_{2} \in m D\left(f^{-1}(U)\right)$ such that the common vanishing set of $s_{1}$ and $s_{2}$ has codimension 2 in $f^{-1}(U)$. Now suppose these sections have poles of order $m_{1}$ and $m_{2}$ along the divisor $\Delta=X \backslash f^{-1}(U)$. Note that $\Delta$ pulls back from a divisor on $S$, so its class belongs to the subspace $f^{*}\left(N^{1}(S)\right) \subset N^{1}(X)$. Since $N^{1}(S)$ is spanned by the classes of ample divisors, we can write (modulo numerically trivial divisors) $\Delta=\sum_{k} n_{k} f^{*}\left(A_{k}\right)$ where the $n_{k}$ are integers (not necessarily positive) and the $A_{k}$ are ample divisors on $S$. Now multiplying $s_{1}$ and $s_{2}$ by appropriate powers of a section $\sigma$ of a line bundle numerically equivalent to $\sum_{k} n_{k} f^{*}\left(A_{k}\right)$ which satisfies $\sigma^{-1}(0)=\Delta$ (as sets), we get global sections $\sigma_{1}$ and $\sigma_{2}$ of line bundles numerically equivalent to $m D+m_{1}\left(\sum_{k} n_{k} f^{*}\left(A_{k}\right)\right)$ and $m D+m_{2}\left(\sum_{k} n_{k} f^{*}\left(A_{k}\right)\right)$, and these sections do not vanish along $\Delta$. Moreover, the restrictions of $\sigma_{1}$ and $\sigma_{2}$ to $f^{-1}(U)$ have the same vanishing sets as $s_{1}$ and $s_{2}$, so we conclude that the common vanishing set of $\sigma_{1}$ 
and $\sigma_{2}$ has codimension 2 in $X$. Finally choose a positive integer $n$ sufficiently large so that $n-m_{1} n_{k}$ and $n-m_{2} n_{k}$ are positive for all $k$, and choose sections $\tau_{1}$ and $\tau_{2}$ of the ample line bundles $\sum_{k}\left(n-m_{1} n_{k}\right) f^{*}\left(A_{k}\right)$ and $\sum_{k}\left(n-m_{1} n_{k}\right) f^{*}\left(A_{k}\right)$ whose common vanishing set has codimension at least 2 and such that the common vanishing set of $\tau_{i}$ and $\sigma_{j}$ has codimension 2 for $i \neq j$. Then $\sigma_{1} \tau_{1}$ and $\sigma_{2} \tau_{2}$ are sections of a line bundle numerically equivalent to $m D+n\left(\sum_{k} f^{*}\left(A_{k}\right)\right)$ whose common vanishing set has codimension at least 2 . Therefore the class $m D+n\left(\sum_{k} f^{*}\left(A_{k}\right)\right)$ is movable.

Now suppose $D$ is any Cartier divisor effective over $S$. By definition this means there is an open set $U \subset S$ and a nonzero section $s$ of $D\left(f^{-1}(U)\right)$. Suppose that $s$ has a pole of order $m$ along the divisor $\Delta=X \backslash f^{-1}(U)$. As above, multiplying $s$ by an appropriate section of a bundle numerically equivalent to $\sum_{k} n_{k} f^{*}\left(A_{k}\right)$ we get a global section $\sigma$ of a bundle numerically equivalent to $D+\sum_{k} n_{k} f^{*}\left(A_{k}\right)$. Therefore $D+\sum_{k} n_{k} f^{*}\left(A_{k}\right)$ is effective, so the map $B^{e}(X) \rightarrow B^{e}(X / S)$ from the effective cone to the relative effective cone is surjective.

Finally suppose $[D] \in \overline{M(X / S)}^{e}$ is the image of a Cartier divisor $D$. We have shown that there are classes $D_{1}=m D+n\left(\sum_{k} f^{*}\left(A_{k}\right)\right) \in \overline{M(X)}$ and $D_{2}=D+\sum_{k} n_{k} f^{*}\left(A_{k}\right) \in B^{e}(X)$. So put $D_{3}=m D+\sum_{k} \nu_{k} f^{*}\left(A_{k}\right)$, where $\nu_{k}=\max \left(n, m n_{k}\right)$. This gives

$$
D_{1}+\sum_{k}\left(\nu_{k}-n\right) f^{*}\left(A_{k}\right)=D_{3}=m D_{2}+\sum_{k}\left(\nu_{k}-m n_{k}\right) f^{*}\left(A_{k}\right) .
$$

For any $r \geq 0$ and any ample divisor $A$ on $S$, the class $r f^{*}(A)$ is in the intersection $B^{e}(X) \cap \overline{M(X)}$, so the left-hand side of the displayed equation is an element of $\overline{M(X)}$ and the right-hand side is an element of $B^{e}(X)$. Therefore $D_{3}$ is an element of the intersection $B^{e}(X) \cap \overline{M(X)}=\overline{M(X)} e^{e}$. So every class $[D] \in \overline{M(X / S)}^{e}$ which is the image of a Cartier divisor class is in the image of the cone $\overline{M(X)}^{e}$, as required.

Having described the relevant cones, we analyse the action of the pseudoautomorphism group on them. First we state a result of Kawamata [11, Lemma 3.5] which shows that, passing to a suitable quotient, the MordellWeil group of our elliptic fibration acts as a group of translations.

Theorem 4.8 (Kawamata). The group $\operatorname{Pic}^{0}\left(X_{\eta}\right)$ acts properly discontinuously on the affine subquotient space $W(X / S)=\left\{z \in N^{1}(X / S) / V(X / S)\right.$ : $z \cdot F=1\}$ as a group of translations, with fundamental domain a rational polyhedron. 
We use this theorem together with our description of $\overline{M(X / S)}^{e}$ to find a rational polyhedral cone whose translates by the Mordell-Weil group cover the relative movable cone:

Lemma 4.9. There is a rational polyhedral subcone $K$ of $\overline{M(X / S)}^{e}$ such that $\operatorname{Pic}^{0}\left(X_{\eta}\right) \cdot K=\overline{M(X / S)}^{e}$.

Proof. Let $W^{\prime}(X / S)$ denote the affine subspace $\left\{y \in N^{1}(X / S): y \cdot F=1\right\}$ and denote by $q$ the quotient map $W^{\prime}(X / S) \rightarrow W(X / S)$. By definition of the quotient action of $\operatorname{Pic}^{0}\left(X_{\eta}\right)$, for any $\phi \in \operatorname{Pic}^{0}\left(X_{\eta}\right)$ and $x \in N^{1}(X / S)$ we have $\phi(q(x))=q(\phi(x))$. By Theorem 4.8 the action of $\operatorname{Pic}^{0}\left(X_{\eta}\right)$ on $W(X / S)$ has fundamental domain a rational polyhedron $\Pi$, and hence for the action on $W^{\prime}(X / S)$ we have $\operatorname{Pic}^{0}\left(X_{\eta}\right) \cdot q^{-1}(\Pi)=W(X / S)$. Since the action of $\operatorname{Pic}^{0}\left(X_{\eta}\right)$ preserves the relative effective movable cone, we can intersect with that cone on both sides to get $\operatorname{Pic}^{0}\left(X_{\eta}\right) \cdot\left(q^{-1}(\Pi) \cap \overline{M(X / S)}^{e}\right)=\overline{M(X / S)}^{e} \cap$ $W(X / S)$. Finally since $\operatorname{Pic}^{0}\left(X_{\eta}\right)$ acts linearly we can multiply on both sides by positive scalars to get $\operatorname{Pic}^{0}\left(X_{\eta}\right) \cdot \mathbf{R}_{+}\left(q^{-1}(\Pi) \cap \overline{M(X / S)}{ }^{e}\right)=\overline{M(X / S)}{ }^{e}$. (Here we use the fact that every ray of $\overline{M(X / S)})^{e}$ intersects $W(X / S)$, which follows from Theorem 4.6.) So taking $K=\mathbf{R}_{+}\left(q^{-1}(\Pi) \cap \overline{M(X / S)}{ }^{e}\right)$ it remains to show that $q^{-1}(\Pi) \cap \overline{M(X / S)}$ is a rational polyhedron in $W^{\prime}(X / S)$. Since $\Pi$ is a rational polyhedron and by Theorem 4.6 the cone $\overline{M(X / S)} e^{e}$ is defined by a finite set of inequalities, we need to show that $q^{-1}(\Pi) \cap$ $\overline{M(X / S)}$ is bounded. Choosing a section $s$ of $q$ we can write $W^{\prime}(X / S)=$ $V(X / S)+\operatorname{im} s$. Let $\Pi^{\prime}$ denote the polyhedron $s(\Pi)$ : then $q^{-1}(\Pi)=V(X / S)$ $+\Pi^{\prime} \subset W^{\prime}(X / S)$. So suppose a vector $v+s$ with $v \in V(X / S)$ and $s \in \Pi^{\prime}$ belongs to $q^{-1}(\Pi) \cap \overline{M(X / S)}^{e}$. By Theorem 4.6 the intersection numbers $(v+s) \cdot F_{i}$ must be nonnegative for all $i$. Now $s \cdot F_{i}$ is bounded for $s \in \Pi^{\prime}$ by compactness of $\Pi^{\prime}$, so $v \cdot F_{i}$ is bounded below for all $i$. Now recall that for any member $\mathcal{D}_{p}$ of our partition we have an expression $\sum_{j \in \mathcal{D}_{p}} m_{j} F_{j}=F$ for some positive integers $m_{j}$. Therefore $v \cdot m_{i} F_{i}=-\sum_{j \in \mathcal{D}_{p}, j \neq i} v \cdot m_{j} F_{j}$ for any $v \in V(X / S)$, so $v \cdot F_{i}$ is bounded above and below. Now fix a basis for $V(X / S)$ consisting of the classes in $N^{1}(X / S)$ of some of the vertical divisors $D_{i}$ : relabelling, we can call these basis elements $\left[D_{1}\right], \ldots,\left[D_{v}\right]$. For a vector $v=\sum_{i=1}^{v} a_{i}\left[D_{i}\right]$ we can solve for the coefficients $a_{i}$ in terms of the intersection numbers $v \cdot F_{i}$, because by Corollary 4.5 the dual of $V(X / S)$ is spanned by the $F_{i}$. Therefore the coefficients of $v$ are bounded. Since $\Pi^{\prime}$ is compact, we conclude that the subset $q^{-1}(\Pi) \cap \overline{M(X / S)}^{e}$ is bounded, hence rational polyhedral, as required. 
Now we complete the proof of our main finiteness result for the movable cone. The final step is to lift the cone $K$ from the previous lemma to a cone in $\overline{M(X)}{ }^{e}$ which has the properties we want.

Theorem 4.10. There exists a rational polyhedral cone $U \subset \overline{M(X)}^{e}$ with the following properties:

(1) $\operatorname{Pic}^{0}\left(X_{\eta}\right) \cdot U$ intersects the interior of every nef cone in $\overline{M(X)}{ }^{e}$.

(2) $U$ is contained in a union $\cup_{i=1, \ldots, n}{\overline{A\left(X_{i}\right)}}^{e}$ of finitely many nef cones in $\overline{M(X)}$.

Proof. The first step is to choose a rational polyhedral cone $K_{0}$ in $\overline{M(X)}{ }^{e}$ which maps onto the cone $K$ from the previous lemma. This is possible by Lemma 4.7. More precisely, for each extremal ray $R_{i}$ of the cone $K$, choose a integral vector $w_{i}$ spanning $R_{i}$, and a Cartier divisor class $D_{i} \in \overline{M(X)}{ }^{e}$ such that $p\left(v_{i}\right)=w_{i}$. This gives a set $\left\{D_{1}, \ldots, D_{p}\right\}$ of vectors in $\overline{M(X)} e^{e}$ spanning a cone which surjects onto $K$. Define $K_{0}$ to be the cone spanned by the set $\left\{D_{1}, \ldots, D_{p}\right\}$.

In choosing generators of the cone $K_{0}$, we are free to replace $D_{i}$ by $D_{i}+$ $\Delta$, where $\Delta$ is any element of $T(X / S)$, as long as the resulting vector still lies in $\overline{M(X)}{ }^{e}$. We can use this to choose generators $D_{1}, \ldots, D_{n}$ lying in the big cone. I claim that if $D \in \overline{M(X)}^{e}$ is a class not in the subspace $T(X / S)$, and $A$ is a general very ample divisor on $S$, then $D+f^{*}(A)$ is big. We use the numerical criterion for bigness of nef divisors [14, Proposition 2.61]: if $N$ is a nef divisor on a $k$-dimensional projective variety $Y$, then $N$ is big if and only if $N^{k}>0$. Choose an SQM $X^{\prime}$ of $X$ on which $D$ is nef; this implies that $D+f^{*}(A)$ is also nef for any ample $A$ on $S$. So consider the self-intersection number $\left(D+f^{*}(A)\right)^{3}=D^{3}+\left(f^{*}(A)\right)^{3}+3 D^{2} \cdot f^{*}(A)+3 D \cdot\left(f^{*}(A)\right)^{2}$. The first 2 terms are nonnegative since $D$ and $f^{*}(A)$ are nef, and the third term is nonnegative since $D^{2} \in \overline{\operatorname{Curv}\left(X^{\prime}\right)}$ and $f^{*}(A)$ is nef. I claim the last term must be strictly positive. If we choose $A$ to be a general very ample divisor on $S$, then $A^{2}$ is a union of $r>0$ points on $S$, and $f^{*}(A)^{2}$ is a union of $r$ general fibres of $f$. So $D \cdot\left(f^{*}(A)\right)^{2}=0$ if and only if $D \cdot F=0$ for $F$ the class of a fibre of $f$, which (since $D$ is nef) implies $D \cdot C=0$ for any fibral curve of $f$. But then $D$ belongs to $T\left(X^{\prime} / S\right)$, which by Lemma 4.2 equals $T(X / S)$. This contradicts our assumption on $D$. We conclude that we can add suitable pullbacks of very ample divisors on $S$ to our generators $D_{i}$ to ensure they are big. The benefit of this change is the following: by Theorem 3.2, the decomposition of $\overline{M(X)}^{e}$ into nef cones is now locally finite near the cone $K_{0}$. In particular $K_{0}$ is contained in a union $\cup_{i=1, \ldots, n}{\overline{A\left(X_{i}\right)}}^{e}$ of finitely many nef cones of SQMs of $X$. 
Now choose an SQM $X^{\prime}$ of $X$ and a Cartier divisor $D$ in the ample cone $A\left(X^{\prime}\right)$. By Lemma 4.9 there exists a divisor $D_{0}$ in $K_{0}$ and an element $\phi \in \operatorname{Pic}^{0}\left(X_{\eta}\right)$ such that $\phi_{*}\left(\left[D_{0}\right]\right)=[D]$ in $N^{1}(X / S)$. Therefore in $N^{1}(X)$ we have $\phi_{*}\left(D_{0}\right)=D+\Delta$, where $\Delta$ is a Cartier divisor in $T(X / S)$. From this expression we cannot conclude that $\phi_{*}\left(D_{0}\right)$ is ample on $X^{\prime}$, so the cone $K_{0}$ is not big enough for our purposes.

To remedy this, fix an ample Cartier divisor class $A$ on $S$. For any $m$ the divisor $f^{*}(m A)$ is fixed by $\operatorname{Pic}^{0}\left(X_{\eta}\right)$, so from the expression above we get $\phi_{*}\left(D_{0}+f^{*}(m A)\right)=D+\Delta+f^{*}(m A)$. Now $D$ is ample on $X^{\prime}$ and $\Delta$ is numerically trivial on fibral curves, therefore $D+\Delta$ is ample over $S$. But then by [14, Proposition 1.45] $D+\Delta+f^{*}(m A)=\phi_{*}\left(D_{0}+f^{*}(m A)\right)$ is ample on $X^{\prime}$ for $m$ a sufficiently large positive integer.

Now define $U$ to be the cone spanned by $K_{0}$ and $f^{*}(A)$. By the previous paragraph, $\operatorname{Pic}^{0}\left(X_{\eta}\right) \cdot U$ intersects the interior of every nef cone inside $\overline{M(X)}{ }^{e}$. It remains to show that $U$ is contained in a union of finitely many nef cones. To see this, observe that $f^{*}(A)$ belongs to every nef cone in $\overline{M(X)}{ }^{e}$ since it is semiample on any SQM of $X$. Therefore $U$ is contained in the same finite union $\cup_{i=1, \ldots, n} \overline{A\left(X_{i}\right)}{ }^{e}$ of nef cones as $K_{0}$.

Corollary 4.11. Suppose $X$ satisfies Assumption 1, and in addition any $S Q M X^{\prime}$ of $X$ has rational polyhedral nef cone. Then the Cone Conjecture holds for $X$.

Proof. By the additional hypothesis, the union $\cup_{i=1, \ldots, n} \overline{A\left(X_{i}\right)}{ }^{e}$ is a rational polyhedral cone whose translates intersect the interior of every nef cone. But since this is a union of effective nef cones, the union of its translates is also a union of effective nef cones, hence must be the entire movable cone. A theorem of Looijenga [16, Application 4.15] then says that the existence of such a cone implies that $\overline{M(X)}{ }^{e}$ has a rational polyhedral fundamental domain for the action of any of the groups $\operatorname{Pic}^{0}\left(X_{\eta}\right), \operatorname{PsAut}(X / S)$, or $\operatorname{PsAut}(X, \Delta)$.

In practice, the condition on the nef cones of all SQMs of a given variety may be difficult to check. However, it has been verified in at least one nontrivial class of examples, which we now describe.

Example 4.12. Let $X$ be the blowup of $\mathbf{P}^{3}$ in the 8 basepoints of a general net of quadrics. In this case, the anticanonical contraction morphism is given by the line bundle $-\frac{1}{2} K_{X}$ and has base $\mathbf{P}^{2}$. The elliptic fibration $X \rightarrow \mathbf{P}^{2}$ has exactly $28=\left(\begin{array}{l}8 \\ 2\end{array}\right)$ reducible fibres, corresponding to the set of lines through 2 of the 8 basepoints of the net. Each reducible fibre is a union $C_{1} \cup C_{2}$ of 2 rational curves meeting transversely in 2 points, in other words type $I_{2}$ in 
Kodaira's classification. (This example can therefore be regarded as a global version of Kawamata's example [11, Example 3.8] in which one considers a versal deformation of an $I_{2}$ fibre.) The SQMs of $X$ are sequences of flops in which we first flop a component $C_{1}$ of a reducible fibre, then flop the proper transform $C_{2}^{\prime}$ of $C_{2}$, then flop the proper transform $C_{1}^{\prime \prime}$ of the other component of the fibre containing $C_{2}^{\prime}$, and so on. (More generally one can have such a sequence of flops for each fibre, but these sequences do not interact.) By explicitly computing the action of the Mordell-Weil group on certain movable classes, one can prove in this case that all the resulting SQMs have rational polyhedral nef cone [23, Theorem 3.5]. As explained above, the full movable cone conjecture therefore holds for $X$.

\section{The nef cone conjecture}

In this section we collect some results which give evidence for the nef cone conjecture for our class of varieties. First we observe that Kawamata's result on the relative nef cone implies that the $K$-trivial part of the cone of curves obeys the conjecture, and then we prove finiteness of most types (in the sense of Mori's list) of divisorial $K$-negative extremal rays. In a slightly different direction, in the smooth case we use the classification results of Bauer-Peternell for 3-folds with nef anticanonical bundle to show that if $X$ is smooth and not rationally connected and $-K_{X}$ has Iitaka dimension 2 , then the nef cone conjecture again holds for $X$.

As always, we assume throughout this section that $X$ is a $\mathbf{Q}$-factorial terminal Gorenstein 3 -fold with $-K_{X}$ semiample of positive Iitaka dimension. The main result (Theorem 5.6) requires the stronger assumption that $X$ is smooth and $-K_{X}$ has Iitaka dimension 2 .

Proposition 5.1. $\overline{\operatorname{Curv}(X)} \cap K^{\perp}=\overline{\operatorname{Curv}(X / S)}$.

Proof. The inclusion $\overline{\operatorname{Curv}(X / S)} \subset \overline{\operatorname{Curv}(X)} \cap K^{\perp}$ is immediate since $f$ : $X \rightarrow S$ is given by a power of $-K_{X}$; it remains to prove the reverse inclusion. Suppose it does not hold: then we can find a point $x \in \overline{\operatorname{Curv}(X)} \cap K^{\perp}$ which does not belong to $\overline{\operatorname{Curv}(X / S)}$. Choose a rational class $H \in N^{1}(X)$ such that $H$ is strictly positive on $\overline{\operatorname{Curv}(X / S)} \backslash\{0\}$, but $H \cdot x<0$. By the Kleiman condition, $H$ is ample over $S$. By [14, Proposition 1.45], if $A$ is any ample class on $S$, then $H+f^{*}(n A)$ is ample on $X$ for $n$ a sufficiently large natural number.

Now the morphism $f: X \rightarrow S$ is given by $-m K_{X}$ for some positive integer $m$, so by the contraction theorem there is an ample line bundle $A$ on 
$S$ such that $f^{*}(A)=-m K_{X}$. Putting this together with the previous paragraph, we see that $H-n K_{X}$ is ample on $X$ for $n$ a sufficiently large and divisible positive integer. But since by assumption $x$ is a point in $K_{X}^{\perp}$, we have $\left(H-n K_{X}\right) \cdot x=H \cdot x<0$, which since $H-n K_{X}$ is ample contradicts the assumption $x \in \overline{\operatorname{Curv}(X)}$.

Corollary 5.2. $\overline{\operatorname{Curv}(X)}$ has only finitely many extremal rays in $K^{\perp}$ corresponding to divisorial contractions or fibre space structures, up to the action of $\operatorname{Aut}(X / S)$.

Proof. By the previous lemma, an extremal ray of $\overline{\operatorname{Curv}(X)}$ lying in $K^{\perp}$ must be an extremal ray of $\overline{\operatorname{Curv}(X / S)}$; moreover, if such an extremal ray corresponds to a contraction morphism, then that contraction is over $S$. Now Kawamata's theorem on the relative nef cone [11, Theorem 3.6, Theorem 4.4] shows that the number of contractions over $S$ is finite up to the action of $\operatorname{Aut}(X / S)$.

Next we consider the $K$-negative extremal rays of $\overline{\operatorname{Curv}(X)}$. By the cone theorem any such ray can be contracted, and so corresponds either to a Mori fibre space structure of $X$ or to a divisorial contraction of one of the 5 types on Mori's list (Theorem 2.1). The next result shows that with the exception of one type, the divisorial contractions of $X$ must always be finite in number.

Proposition 5.3. The number of $K$-negative extremal contractions of $X$ of types 2, 3, 4 and 5 on Mori's list is finite.

Proof. I claim that if $\phi: X \rightarrow Y$ is a $K$-negative extremal contraction of type 2, 3, 4 or 5 on Mori's list, the exceptional divisor $D_{\phi}$ of $\phi$ must be disjoint from the exceptional divisor of any other $K$-negative extremal contraction. Given this, the proposition follows, since otherwise we could find a morphism $f: X \rightarrow Z$ of projective varieties contracting an arbitrary number (in particular more than $\rho(X)-1$ ) of prime divisors on $X$ whose classes in $N^{1}(X)$ are linearly independent.

To prove the claim, suppose $\phi: X \rightarrow Y$ is of type $2,3,4$ or 5 , and $\psi: X \rightarrow Z$ is any $K$-negative extremal contraction. If $D_{\phi}$ is not disjoint from the exceptional divisor $D_{\psi}$ of $\psi$, then they intersect in a 1-dimensional cycle supported on a curve $C$. So $\psi$ contracts the curve $C \subset D_{\phi}$. But the description in Theorem 2.1 of the exceptional divisor $D_{\phi}$ tells us that for $\phi$ of type $2,3,4$ or 5 , all curves in $D_{\phi}$ are numerically proportional in $N_{1}(X)$, so $\psi$ must contract all of $D_{\phi}$. Since the exceptional divisor of $\psi$ is prime, we conclude that $\psi=\phi$. 
To summarise, we have shown finiteness up to automorphisms of the number of $K$-trivial extremal contractions, and finiteness of the number of divisorial $K$-negative extremal contractions of all but one type on Mori's list. To complete the proof of the nef cone conjecture for the given class of varieties, it remains to show finiteness up to automorphisms of the set of divisorial contractions of type 1 on Mori's list and the set of $K$-negative fibre space structures of $X$. I am unable to do this at present.

We conclude by mentioning the classification results of Bauer-Peternell for smooth 3 -folds with $-K_{X}$ nef, and showing how these reduce the nef cone conjecture to the rationally connected case. We should point out that the theorem below is only a small part of Bauer-Peternell's results, which give information about all smooth 3 -folds with $-K_{X}$ nef and numerically nontrivial. See [1] for details. From now on, we consider only smooth $X$ with $-K_{X}$ semiample of Iitaka dimension 2 .

Theorem 5.4 (Bauer-Peternell). Suppose that $X$ is a smooth 3-fold with $-K_{X}$ semiample of Iitaka dimension 2. Then one of the following holds:

(1) $X$ is rationally connected;

(2) $X \cong_{f} S \times B$ with $S$ a del Pezzo surface, $B$ an elliptic curve;

(3) $X \cong_{f} \mathbf{P}(E) \times B$ with $E$ a rank-2 bundle over an elliptic curve $A$, and $B$ another elliptic curve.

Here the symbol $\cong_{f}$ means that some finite etale cover of $X$ is isomorphic to the given variety.

Bauer-Peternell give analogous results in the case $-K_{X}$ of Iitaka dimension 1 , but I was not able to apply these results to the cone conjecture.

Corollary 5.5. For $X$ as in Theorem 5.4, if $X$ is not rationally connected, then $\overline{C u r v(X)}$ has finitely many $K$-negative extremal rays.

Proof. By Theorem 5.4 if $X$ is not rationally connected there is a finite etale cover $\pi: Y \rightarrow X$ such that $Y$ has the one of the two product structures listed. I claim that the set of $K$-negative extremal rays of $\overline{\operatorname{Curv}(X)}$ can be identified naturally with a subset of the $K$-negative extremal rays of $\overline{\operatorname{Curv}(Y)}$, and that the latter set is finite.

First note that since $\pi$ is etale, the sheaf of relative differentials $\Omega_{Y / X}$ is zero, so the exact sequence $\pi^{*} \Omega_{X} \rightarrow \Omega_{Y} \rightarrow \Omega_{Y / X} \rightarrow 0$ implies that $\Omega_{Y}=$ $\pi^{*} \Omega_{X}$, which in turn gives $K_{Y}=\pi^{*} K_{X}$. In particular, if $C$ is any curve 
on $X$, we have $K_{X} \cdot C<0$ if and only if $K_{Y} \cdot \pi^{-1}(C)<0$. Now suppose $R$ is a $K$-negative extremal ray of $\overline{\operatorname{Curv}(X)}$. By the cone theorem, there is a semiample line bundle $L$ on $X$ such that $\overline{\operatorname{Curv}(X)} \cap L^{\perp}=R$. The pullback $\pi^{*} L$ is again semiample, and clearly contracts no curves except those whose image on $X$ have class in the ray $R$. This proves the first claim, that the set of $K$-negative extremal rays of $\overline{\operatorname{Curv}(X)}$ can be identified with a subset of the $K$-negative extremal rays of $\overline{\operatorname{Curv}(Y)}$. It remains to show that $\overline{\operatorname{Curv}(Y)}$ has only finitely many $K$-negative extremal rays. This follows easily from the product structure of $Y$, as we now explain.

Case 1: here $Y=S \times B$, with $S$ a del Pezzo surface, $B$ an elliptic curve. Denote the projections of $Y$ onto its two factors by $p r_{1}$ and $p r_{2}$. Suppose $R$ is a $K$-negative extremal ray of $\overline{\operatorname{Curv}(Y)}$. Then $R$ is spanned by the class of a rational curve $C$. There cannot be a nonconstant morphism $C \rightarrow B$ since $B$ has genus 1, so $C$ must be contained in a surface $\operatorname{pr}_{2}^{-1}(p) \cong S$ inside $Y$, where $p \in B$ is a point. Therefore the ray $R$ must belong to the subcone $\overline{\operatorname{Curv}(Y / B)} \subset \overline{\operatorname{Curv}(Y)}$. Since $Y$ is a product $S \times B$, we have $\overline{\operatorname{Curv}(Y / B)} \cong$ $\overline{\operatorname{Curv}(S)}$, which is rational polyhedral since $S$ is del Pezzo. So there are only finitely many possibilities for $R$.

Case 2: here $Y=\mathbf{P}(E) \times B$, with $E$ a rank-2 bundle on an elliptic curve $A$, and $B$ another elliptic curve. As above, any rational curve on $Y$ must project to a point on $B$, so any $K$-negative extremal ray $R \subset \overline{\operatorname{Curv}(Y)}$ must belong to the subcone $\overline{\operatorname{Curv}(Y / B)}$. Again this is just the cone $\overline{\operatorname{Curv}(\mathbf{P}(E))}$. Any ruled surface has Picard number 2, so its closed cone of curves has exactly 2 extremal rays.

By Proposition 5.2 the set of extremal rays in $K^{\perp}$ corresponding to birational contractions or fibre space structures is in any case finite up to the action of $\operatorname{Aut}(X / S)$, so we can put these results together to get the following.

Theorem 5.6. Suppose $X$ is a smooth 3-fold with $-K_{X}$ semiample of Iitaka dimension 2 , and $X$ is not rationally connected. Then $\overline{\operatorname{Curv}(X)}$ has finitely many extremal rays corresponding to birational contractions or fibre space structures, up to the action of $\operatorname{Aut}(X)$.

\section{Acknowledgements}

Thanks to Burt Totaro for many helpful discussions, and to the referee for a number of suggestions. 


\section{References}

[1] T. Bauer and T. Peternell, Nef reduction and anticanonical bundles. Asian J. Math., 8 (2004), no. 2, 315-352.

[2] C. Birkar, P. Cascini, C. Hacon and J. McKernan, Existence of minimal models for varieties of log general type. J. Amer. Math. Soc., 23 (2010), no. 2, 405-468.

[3] C. Borcea, On desingularized Horrocks-Mumford quintics. J. Reine Angew. Math., 421 (1991), 23-41.

[4] S. Cutkosky, Elementary contractions of Gorenstein threefolds. Math. Ann., 280 (1988), no. 3, 521-525.

[5] R. Friedman and D. Morrison (eds.), The birational geometry of degenerations. Birkhäuser (1983).

[6] M. Fryers, The movable fan of the Horrocks-Mumford quintic. arXiv: math/0102055.

[7] A. Grassi and D. Morrison, Automorphisms and the Kähler cone of certain Calabi-Yau manifolds. Duke Math. J., 71 (1993), no. 3, 831838.

[8] R. Hartshorne, Algebraic Geometry. Springer (1977).

[9] Y. Kawamata, Crepant blowing-up of 3-dimensional canonical singularities and its application to degenerations of surfaces. Ann. of Math., 127 (1988), 93-163.

[10] Y. Kawamata, Termination of log flips for algebraic 3-folds. Internat. J. Math., 3 (1992), 653-659.

[11] Y. Kawamata, On the cone of divisors of Calabi-Yau fiber spaces. Internat. J. Math., 8 (1997), 665-687.

[12] Y. Kawamata and K. Matsuki, The number of the minimal models for a 3-fold of general type is finite. Math. Ann., 276 (1987), 595-598.

[13] J. Kollár, Flops. Nagoya Math. J., 113 (1989), 15-36.

[14] J. Kollár and S. Mori, Birational Geometry of Algebraic Varieties. Cambridge University Press (1998).

[15] R. Lazarsfeld, Positivity in Algebraic Geometry. I. Springer (2004). 
[16] E. Looijenga, Discrete automorphism groups of convex cones of finite type. Compos. Math., to appear.

[17] E. Markman, A survey of Torelli and monodromy results for holomorphic-symplectic varieties. Complex and differential geometry, 257-322. Springer (2011).

[18] E. Markman and K. Yoshioka, A proof of the Kawamata-Morrison Cone Conjecture for holomorphic symplectic varieties of $K 3^{[n]}$ or generalized Kummer deformation type. arXiv:1402.2049.

[19] S. Mori, Threefolds whose canonical bundles are not numerically effective. Ann. of Math., 116 (1982), 133-176.

[20] D. Morrison, Compactifications of moduli spaces inspired by mirror symmetry. Journées de géométrie algébrique d'Orsay (Orsay, 1992). Astérisque, 218 (1993), 243-271.

[21] Y. Namikawa, Periods of Enriques surfaces. Math. Ann., 270 (1985), 201-222.

[22] K. Oguiso, On the finiteness of fiber-space structures on a Calabi-Yau 3-fold. J. Math. Sci., 106 (2001), 3320-3335.

[23] A. Prendergast-Smith, The cone conjecture for some rational elliptic threefolds. Math. Z., 272 (2012), 589-605.

[24] A. Prendergast-Smith, The cone conjecture for abelian varieties. J. Math. Sci. Univ. Tokyo, 19 (2012), 243-261.

[25] M. Reid, Minimal models of canonical 3-folds. Algebraic varieties and analytic varieties, (Tokyo, 1981), 131-180.

[26] H. Sterk, Finiteness results for algebraic K3 surfaces. Math. Z., 189 (1985), 507-513.

[27] B. Szendröi, Some finiteness results for Calabi-Yau threefolds. J. London Math Soc., 60 (1999), 689-699.

[28] B. Totaro, Hilbert's fourteenth problem over finite fields, and a conjecture on the cone of curves. Compos. Math., 144 (2008), no. 5, 11761198.

[29] B. Totaro, The cone conjecture for Calabi-Yau pairs in dimension two. Duke Math. J., 154 (2010), 241-263. 
[30] H. Uehara, Calabi-Yau threefolds with infinitely many divisorial contractions. J. Math. Kyoto Univ., 44 (2004), 99-118.

[31] P. M. H. Wilson, Minimal models of Calabi-Yau threefolds. Classification of algebraic varieties, (L'Aquila, 1992), 403-410.

Department of Mathematical Sciences, Loughborough University

LE11 3TU, United KingDom

E-mail address: A.Prendergast-Smith@lboro.ac.uk

ReCeived April 15, 2013 